\title{
Determining the role of the underlying orbital-dependence of PBE0-DH and PBE-QIDH double-hybrid density functionals
}

J. C. Sancho-García ${ }^{1 *}$ A. J. Pérez-Jiménez ${ }^{1}$, M. Savarese ${ }^{2}$, E. Brémond ${ }^{2}$, and C. Adamo ${ }^{2,3}$

${ }^{1}$ Departamento de Química Física, Universidad de Alicante, E-03080 Alicante, Spain

${ }^{2}$ CompuNet, Istituto Italiano di Tecnologia, via Morego 30, I-16163 Genoa, Italy

${ }^{3}$ Institut de Recherche de Chimie Paris, IRCP CNRS UMR-8247, Chimie ParisTech, École Nationale Superieure de Chimie de Paris, 11 rue P. et M. Curie, F-75231 Paris Cedex 05, France

February 20, 2017

*E-mail: jc.sancho@ua.es 


\begin{abstract}
We study the orbital-dependence of three (parameter-free) doublehybrid density functionals, namely the PBE0-DH, the PBE-QIDH models, and the SOS1-PBE-QIDH spin-opposite-scaled variant of the latter. To do it, we feed all their energy terms with different sets of orbitals obtained previously from self-consistent DFT calculations using several exchange-correlation functionals (eg. PBE, PBE0, PBEH\&H), or directly with HF-PBE orbitals, to see their effect on selected datasets for atomization and reaction energies, the latter proned to marked self-interaction errors. We find that the PBE-QIDH double-hybrid model shows a great consistency, since the best results are always obtained for the set of orbitals corresponding to its hybrid scheme, which prompts us to recommend this model without any other fitting or reparameterization.
\end{abstract}

Key words: double-hybrid density functionals, orbital dependence, densitydriven vs. energy-driven errors, AE6 and SIE11 datasets. 


\section{Introduction}

Density Functional Theory (DFT), virtually in almost all applications, relies on the Kohn-Sham (KS) framework [1], in which one-electron equations are solved leading to the corresponding orbitals and associated eigenvalues. These orbitals are self-consistently obtained under the action of a KS potential, whose components are the functional derivative of all the energy terms, including necessarily the key exchange-correlation effects. Since the exact expression for the exchange-correlation functional $E_{x c}$ is still unknown, we have assisted in last decades to huge advances from the derivation of more accurate mathematical forms. One of the latest family of these approximations, dubbed as Double-Hybrid (DH) density functionals, are becoming increasingly popular [2-5]. Their accuracy is usually higher than the previous categories of existing methods [6]. To understand their ingredients, we generally express them as the following linear combination of energy terms [7]:

$$
E_{x c}^{\mathrm{DH}}[\rho]=\lambda_{x} E_{x}^{\mathrm{EXX}}[\phi]+\left(1-\lambda_{x}\right) E_{x}^{\mathrm{DFA}}[\rho]+\lambda_{c} E_{c}^{\mathrm{PT} 2}\left[\phi, \phi^{\prime}\right]+\left(1-\lambda_{c}\right) E_{c}^{\mathrm{DFA}}[\rho],
$$

with $\lambda_{x}\left(0 \leq \lambda_{x} \leq 1\right)$ and $\lambda_{c}\left(0 \leq \lambda_{c} \leq 1\right)$ being scaling parameters of the different energy terms, with $E_{x}^{\mathrm{DFA}}[\rho]\left(E_{c}^{\mathrm{DFA}}[\rho]\right)$ standing for the semilocal Density Functional Approximation (DFA) selected for exchange (correlation) effects, evaluated with the density $\rho(\mathbf{r})=\sum_{i}\left|\phi_{i}\right|^{2}$ built from the occupied orbitals $\phi_{i}$ obtained self-consistently. We emphasize herein the dependence of the terms in Eq. (1) on the occupied (unoccupied) spin-orbitals, $\left\{\phi_{i}\right\}\left(\left\{\phi_{a}^{\prime}\right\}\right)$ with eigenvalues $\left\{\epsilon_{i}\right\}\left(\left\{\epsilon_{a}\right\}\right)$. This dual dependence, on both occupied and unoccupied orbitals, is probably made more explicit through considering the Exact-like EXchange (EXX) and second-order perturbative (PT2) terms:

$$
E_{x}^{\mathrm{EXX}}[\phi]=-\frac{1}{2} \sum_{i, j}\left\langle\phi_{i} \phi_{j} \mid \phi_{j} \phi_{i}\right\rangle
$$




$$
E_{c}^{\mathrm{PT} 2}\left[\phi, \phi^{\prime}\right]=-\frac{1}{4} \sum_{i, j} \sum_{a, b} \frac{\left|\left\langle\phi_{i} \phi_{j}|| \phi_{a}^{\prime} \phi_{b}^{\prime}\right\rangle\right|^{2}}{\epsilon_{a}+\epsilon_{b}-\epsilon_{i}-\epsilon_{j}},
$$

with the two-electron integrals being expressed using a short notation as $\langle i j|| a b\rangle=\langle i j \mid a b\rangle-\langle i j \mid b a\rangle$. Note that we neglect here in the $E_{c}^{\mathrm{PT} 2}$ term the difference between the local KS exchange potential and the non-local exactexchange potential, as it is usually done due to their vanishing influence [8].

One fundamental problem, to which developers are given now due attention, is the orbital-dependence of the above expression, Eq. (1), or in other words, how to generate the best orbitals for each of the newly conceived double-hybrid models. Generally speaking, and starting from the simplest possible case, if $\lambda_{x}=\lambda_{c}=0$ the orbitals are generated by including the contribution of the exchange-correlation potential, $v_{x c}=v_{x}^{\mathrm{DFA}}+v_{c}^{\mathrm{DFA}}$, to the total Kohn-Sham effective potential, $\left(-\frac{1}{2} \nabla_{i}+v_{e f f}(\mathbf{r})\right) \phi_{i}(\mathbf{r})=\epsilon_{i} \phi_{i}(\mathbf{r})$, used to obtain the orbital manifold. In case of having the following combination of scaling parameters $\lambda_{x} \neq 0$ and $\lambda_{c}=0$, ie. a global hybrid density functional in this case, the orbitals are generated also self-consistently under an effective potential, but after considering the $E_{x}^{\mathrm{EXX}}$ term as a functional of the one-particle density matrix, $\rho\left(\mathbf{r}, \mathbf{r}^{\prime}\right)=\sum_{i} \phi_{i}^{\star}(\mathbf{r}) \phi_{i}\left(\mathbf{r}^{\prime}\right)$, which leads to the corresponding (scaled by $\lambda_{x}$ ) non-local contribution to the exchange-correlation potential [9]. Note that none of the expressions entering into a non-hybrid (semilocal) or a hybrid functional depend on the set of virtual orbitals, contrarily to what happens with double-hybrid models $\left(\lambda_{x} \neq 0\right.$ and $\left.\lambda_{c} \neq 0\right)$ through the $E_{c}^{\mathrm{PT} 2}\left[\phi, \phi^{\prime}\right]$ term.

Therefore, when one employs a DH model, the procedure mostly followed up to now disregards the contribution of the perturbative correlation energy to the orbital calculation, and the exchange-correlation potential thus resem- 
bles that for a hybrid model, although with the particular value of $\lambda_{x}$ and $\lambda_{c}$ scaling the respective terms. Once these orbitals are converged, they are used to calculate the $E_{c}^{\mathrm{PT} 2}$ correlation energy which is added a posteriori to the total energy. This procedure is known to work reasonably well except for some difficult (eg. open-shell or symmetry-breaking) cases, as it was also documented before for hybrid density functionals [10-13], where the results can be improved by an orbital-optimized scheme to obtain a set of orbitals built under the influence of the perturbative field too $[14,15]$. Another successful approach circumvents these caveats by using the orbitals generated by a hybrid model (eg. the PBE0 functional $[16,17]$ ) directly into the DH expression, giving rise to the family of $\mathrm{xDH}$ functionals [18-21]. Recent advances in this direction makes also use of the Optimized Effective Potential (OEP) technique [22], including the perturbative contribution to the Kohn-Sham potential $[23,24]$, and exemplifying the interest within the field for further understanding and improved developments related to the orbital-dependence of double-hybrid density functionals.

Therefore, once the density functional expression is chosen, two potential sources of errors in standard calculations coexist: the one due to the approximate density built-in self-consistently along the calculation (ie. the density-driven error) and that due to the limitations of the energy expression by itself (ie. the energy-driven error). We have thus tried to understand the importance of these two errors for recently developed parameter-free doublehybrid models, investigating consequently here their orbital-dependence by varying the set of orbitals used to evaluate the electronic energy. 


\section{Computational details}

We select here the PBE exchange and correlation models [25] for $E_{x}^{\mathrm{DFA}}[\rho]$ and $E_{c}^{\mathrm{DFA}}[\rho]$, and a pair of parameter-free $\mathrm{DH}$ functionals recently developed, PBE0-DH [26] and PBE-QIDH [27], the latter QIDH acronym stands for Quadratic Integrand $\mathrm{DH}$, with the corresponding values of $\lambda_{x}$ and $\lambda_{c}$ gathered in Table 1. Note that the latter model has been also recently made spin-component-scaled [28], the SOS1-PBE-QIDH variant, leading to accurate results still keeping the parameter-free nature of the original PBE-QIDH. The interest in the latter and related variants arises from the reduction of the formal scaling from $O\left(N^{5}\right)$ to $O\left(N^{4}\right), N$ related to the system size, which could be further exploited in future codes [29]. Note that we do not aim at exploring the large variety of existing $\mathrm{DH}$ models, but restrict ourselves to some first-principles models to avoid mixing up parameterization effects (ie. rescaling the values of $\lambda_{x}$ and $\lambda_{c}$ for each set of orbitals used) with the underlying orbital-dependence of any of the models.

All the DH calculations were done with the the ORCA (release 3.0.1) package [30] with tight convergence and integration thresholds. We always employ the very large aug-cc-pVQZ basis set, to reduce basis-set errors as much as possible. We choose the AE6 (atomization energies of small molecules [31,32]) with values updated from explicitly correlated coupledcluster theory [33], and SIE11 (reactions prone to self-interaction errors [34]) datasets, as they are highly representative of challenging systems with a possibly marked dependence on the built-in orbitals used, and employ as metrics for the performance of the methods the Mean Absolute Deviation (MAD) and the Root Mean-Squared Deviation (RMSD) with respect to reference results. 


\section{Results and discussion}

\subsection{Atomization Energies}

We would like first to isolate what we consider the main factor governing the orbital-dependence results for DHs. Taking into account the fact that the $E_{x}^{\mathrm{DFA}}[\rho]$ and $E_{c}^{\mathrm{DFA}}[\rho]$ terms are only slightly affected by the correlation potential (indeed the occupied set of orbitals for individual molecules is found rather similar for different functionals $[35,36])$ we first set $\lambda_{c}=0$ and $\lambda_{x} \neq 0$ to generate the different orbitals in a controlled manner. We will therefore employ in the following a variety of methods to have different effective potentials, ranging from semilocal (ie. PBE, $\lambda_{x}=0$ ) to hybrids (ie. PBE0, $\lambda_{x}=1 / 4$; and adhoc PBEH\&H, $\left.\lambda_{x}=1 / 2\right)$ and to the pure HartreeFock method combined with the corresponding correlation functional (ie. HF-PBE, $\left.\lambda_{x}=1\right)$. Once the orbitals are self-consistently generated in a first step, we use the whole occupied and virtual manifold to obtain the energy of all terms entering into Eq. (1) in a second step. In such a way, once the PBE0-DH, PBE-QIDH, and SOS1-PBE-QIDH model is selected, we may thus disentangle for each of the methods the underlying (if any) orbital-dependence, and then the compromise between energy-driven and density-driven errors [37].

Figure 1 shows the evolution of the MAD (the RMSD behaves similarly) for the AE6 database, as a function of the aforesaid orbital sets. We add the QIDH hybrid orbitals (generated with a value of $\lambda_{x}=3^{-1 / 3} \approx 0.693$ ) to mimic also the corresponding weight when a PBE-QIDH self-consistent calculation is performed. Note that the SOS1-PBE-QIDH method shares with PBE-QIDH the same expression for the exchange part, and that for PBE0$\mathrm{DH}$ the self-consistent calculation corresponds to the PBEH\&H $\left(\lambda_{x}=1 / 2\right)$ 
case so that we don't need to additionally add that point to the graph. It can be immediately seen how the results markedly depend on the set of orbitals used, in agreement with other results from the literature [38], with the MAD values roughly spanning between $5-10 \mathrm{kcal} / \mathrm{mol}$ for PBE0-DH, and between 5-20 for (SOS1-)PBE-QIDH. We observe at first sight a weakest dependence of the PBE0-DH model on the set of orbitals, as well as a parallel behavior between PBE-QIDH and its corresponding SOS1-based version. After performing a splines based fitting of the data, the lowest MAD values are obtained for $\lambda_{x}=0.25, \lambda_{x}=0.69$, and $\lambda_{x}=0.50$, for PBE0-DH, PBEQIDH, and SOS1-PBE-QIDH, respectively. Interestingly, the value of $\lambda_{x}$ for PBE-QIDH almost exactly matches that obtained during the development of the model (see Table 1). Nonetheless, it is also gratifying to observe how the orbitals generated by a hybrid functional, within the range $0.2 \leq \lambda_{x} \leq 0.5$, would lead to reasonable MAD values for all cases.

We have also corroborated that the use of one or another exchangecorrelation functional to generate the orbitals does not bring further differences. If, for instance, we employ now for the PBE-QIDH calculations the $\operatorname{BLYP}\left(\lambda_{x}=0\right)$ or the BH\&HLYP $\left(\lambda_{x}=1 / 2\right)$ functionals, instead of the corresponding $\mathrm{PBE}\left(\lambda_{x}=0\right)$ or $\mathrm{PBEH} \& \mathrm{H}\left(\lambda_{x}=1 / 2\right)$ ones, the MAD differs now respectively by $0.1-0.2 \mathrm{kcal} / \mathrm{mol}$ for both PBE/BLYP or PBEH\&H/BH\&HLYP pair of calculations, which can be thus considered as a negligible influence. The B3LYP results $\left(\lambda_{x}=0.2\right)$ lies also close to those provided by $\operatorname{PBE} 0\left(\lambda_{x}=1 / 4\right)$, as it should be expected due to the marked dependence of the results on the $\lambda_{x}$ of the global hybrid functionals generating the orbitals. These results show the dominant influence of this $\lambda_{x}$ weight, bringing the $E_{x}^{\mathrm{DFA}}[\rho]$ and $E_{c}^{\mathrm{DFA}}[\rho]$ particular forms employed little 
differences.

We try to rationalize now the above results by considering the evolution of the exchange and correlation components entering into the PBE-QIDH model with the $\lambda_{x}$ value used to generate the orbitals. Table 2 lists the energies for the set of atoms included into the AE6 dataset, with the corresponding trends plotted in Figures 2 and 3. The trends observed in Figure 2 indicate that, irrespective of the atom considered, both exchange contributions favour the HF-like orbitals, while the PT2 correlation contribution counteracts this trend, irrespective of the atom considered. On the other hand, the DFA correlation term has a more involved behavior, yielding a minimum around $\lambda_{x} \sim 0.6$ for $\mathrm{C}, \mathrm{Si}$ and $\mathrm{S}$, while for $\mathrm{O}$ it is placed at $\lambda_{x}=1$. The overall trend for the total exchange-correlation contribution is gathered in Figure 3, showing that $\mathrm{C}, \mathrm{O}$, and $\mathrm{S}$ yield minima placed at $\lambda_{x} \sim 0.6-0.7$, while for Si that minimum is found at $\lambda_{x}=1$, mostly due to the PT2 correlation contribution being somewhat lower than expected in that case. Actually, if we compare the atomic correlation energies provided by the PBE-QIDH model, when one feeds the model with the PBE, PBE0, PBEH\&H, or HF-PBE orbitals, with respect to reference values [39], we found that relative errors are reasonably comprised between $19-26 \%$.

The above results evidence that the "optimal" set of orbitals preferred by the exchange-correlation term results from a delicate balance maintained by all four contributions to the exchange-correlation energy. These, in turn, depend both on the coefficients $\lambda_{x}$ and $\lambda_{c}$, (mostly influencing the energydriven error) and on the orbitals (mostly influencing the density-driven error). For the PBE-QIDH model, that "optimal" set of orbitals corresponds to a 
value of $\lambda_{x} \sim 0.7$, which coincides with the value of $\lambda_{x}$ that is actually used to define the model. However, this is not always the case, as the PBE0DH results on the AE6 benchmark indicate, and the self-interaction error reactions analyzed in section 3.3 will also corroborate.

\subsection{One-electron self-interation error}

The one-electron Self-Interaction Error (SIE) is commonly defined by the conditions [40]: (i) $\int d \mathbf{r} \rho_{\alpha}=1$ and $\rho_{\beta}=0$; and (ii) $E_{x}\left[\rho_{\alpha}, \rho_{\beta}=0\right]=$ $-J\left[\rho_{\alpha}, \rho_{\beta}=0\right]$ together with $E_{c}\left[\rho_{i \in \alpha, \beta}\right]=0$, which is not usually translated into the corresponding potentials $v_{x}\left[\rho_{\alpha}, \rho_{\beta}=0\right]=-u\left[\rho_{\alpha}, \rho_{\beta}=0\right]$ and $v_{c}\left[\rho_{i \in \alpha, \beta}\right]=0$. The simplest way to correct it is to include the full amount of EXX, which is by definition an energy and orbital SIE-free scheme, in routine DFT calculations; although it is also known how this scheme precludes the achievement of great accuracy in standard calculations. On the other hand, double-hybrid functionals are expected to have a reduced SIE compared to hybrid models, due to the larger values of $\lambda_{x}$ entering into their formulation (eg. $\lambda_{x}=1 / 2$ for PBE0-DH and $\lambda_{x}=3^{-1 / 3}$ for PBE-QIDH). Independently of this, we would like to check the dependence of the SIE on the orbitals used to feed the energy terms. Taking the PBE-QIDH as an example, the spurious one-electron interaction energy of the $\mathrm{H}$ atom goes from $1.4 \mathrm{kcal} / \mathrm{mol}$ (with PBE orbitals, and thus largely affected by SIE) to $1.2 \mathrm{kcal} / \mathrm{mol}$ (with virtually "SIE-free" HF-PBE orbitals), indicating the almost negligible effect of the orbitals here. Borrowing again the distinction between density-driven and energy-driven errors, the SIE seems to be an energy-driven magnitude, that is, influenced the most by the energy expression considered. 


\subsection{Self-interaction error reactions}

We extend the former analysis to the SIE11 dataset [41] of chemical reactions, whose use has become widely popular in last years to benchmark the N-electron self-interaction error, also known as delocalization error, of den-

sity functionals $[42,43]$. As it was done before for the AE6 dataset, Figure 4 shows the evolution of the MAD (the RMSD behaves similarly again) as a function of the different orbital sets used to feed the PBE0-DH, PBE-QIDH, and SOS1-PBE-QIDH double-hybrid density functionals selected. Compared with the previous results on the AE6 dataset, we would like to emphasize now that: (i) the MAD is less affected upon the use of different molecular orbitals, taking the PBE-QIDH as example the MAD evolves from its highest $(8.7 \mathrm{kcal} / \mathrm{mol}$, with PBE orbitals $)$ to its lowest $(3.4 \mathrm{kcal} / \mathrm{mol}$, with its own QIDH orbitals) value with the corresponding ratio being roughly 2.5 , to be compared with the equivalent value of 4.5 obtained before for the AE6 dataset; (ii) interestingly, the lowest MAD is found exactly at the value for $\lambda_{x}=0.69$ originally derived for the PBE-QIDH model, which confirms the minimal N-electron SIE of the functional irrespectively of the orbitals used; (iii) the PBE0-DH model, keeping a low value for $\lambda_{c}$, is only moderately affected by the use of any set of orbitals, with the HF-PBE set of orbitals providing now its lowest MAD; and (iv) the SOS1-PBE-QIDH version leads to a very low MAD of roughly $3.0 \mathrm{kcal} / \mathrm{mol}$ with the PBE orbitals, apparently the most affected by the SIE, and shows a somehow unexpected evolution, with the self-interaction free HF orbitals giving the highest MAD of $5.5 \mathrm{kcal} / \mathrm{mol}$. 


\section{Conclusions}

The PBE0-DH model, with $\lambda_{x}=1 / 2$ and $\lambda_{c}=1 / 8$, shows for the AE6 and SIE11 databases a less pronounced dependence with respect to the orbital sets used to feed the model, contrarily to what happens with PBE-QIDH for which $\lambda_{x}=3^{-1 / 3}$ and $\lambda_{c}=1 / 3$, which seems to be related with the orbital and eigenvalue dependence of the $\lambda_{c} E_{c}^{\mathrm{PT} 2}$ expression as deduced from Eq. (3). Notwithstanding this, the latter model keeps some advantages, since it leads to the lowest MAD with the orbitals generated by a hybrid model displaying a value of $\lambda_{x} \approx 0.7$, and thus virtually identical to the one introduced originally into its derivation, indicating thus the excellent compromise between energy- and density-driven errors achieved by this model.

Concerning the self-interaction error affecting many relevant chemical properties, the use of the HF-PBE orbitals (almost self-interaction free by definition) does not impact too much on the results with respect to the KS orbitals obtained self-consistently with the default $\lambda_{x}=3^{-1 / 3}$ value of the PBE-QIDH model. Furthermore, the lowest MAD values found for the latter (4.5 and $3.4 \mathrm{kcal} / \mathrm{mol}$ for the AE6 and SIE11 datasets, respectively) are lower than those provided by PBE0-DH, and actually very competitive with other modern double-hybrid models [44].

However, when one attempts to derive a spin-component-based expression from the corresponding double-hybrid density functionals, eg. the SOS1PBE-QIDH variant from the pristine PBE-QIDH, it seems that there is a stronger coupling between the energy expression and the set of orbitals feeding the energy terms, which might justify the need of a reparameterization of the original weights scaling the exchange and correlation energy terms, 
to couple and then minimize both density- and energy-driven errors. That would help to explain the success of expressions like xDH-PBE0, rationalizing thus their great accuracy.

Overall, the PBE-QIDH model arises as a robust and accurate expression, avoiding the use of any other set of orbitals than those self-consistently generated along a standard calculation.

\section{Acknowledgements}

JCSG and AJPJ thank the "Ministerio de Economía y Competitividad" of Spain and the "European Regional Development Fund" through project CTQ2014-55073-P.

\section{References}

[1] Kohn, W.; Sham, L. J. Phys. Rev. A 1965, 140, 1133.

[2] Zhang, I. Y.; Xu, X. Int. Rev. Phys. Chem. 2011, 30, 115.

[3] Sancho-García, J. C.; Adamo, C. Phys. Chem. Chem. Phys. 2013, 15, 14581.

[4] Goerigk, L.; Grimme, S. WIREs Comput. Mol. Sci. 2014, 4, 576.

[5] Schwabe, T. Chemical Modeling, edited by M. Springborg and J.-O. Joswig, 2016, 13, 191.

[6] Brémond, E.; Ciofini, I.; Sancho-García, J. C.; Adamo, C. Acc. Chem. Res. 2016, 49, 1503. 
[7] Grimme, S. J. Chem. Phys. 2006, 124, 034108.

[8] Engel, E. A Primer in Density Functional Theory, edited by C. Fiolhais et al., Springer-Verlag, Belin Heidelberg, 2003.

[9] Seidl, A.; Görling, A.; Vogl, P.; Majewski, J. A.; Levy, M. Phys. Rev. B 1996, 53, 3764 .

[10] Janesko, B. G.; Scuseria, G. E. J. Chem. Phys. 2008, 128, 244112.

[11] Kim, M.-C.; Sim, E.; Burke, K. J. Chem. Phys. 2011, 134, 171103.

[12] Kim, M.-C.; Sim, E.; Burke, K. Phys. Rev. Lett. 2013, 111, 073003.

[13] Kim, M.-C.; Park, H.; Sim, E.; Burke, K. J. Phys. Chem. Lett. 2015, 6,3802 .

[14] Peverati, R.; Head-Gordon, M. J. Chem. Phys. 2013, 139, 024110.

[15] Sancho-García, J. C.; Pérez-Jiménez, A. J.; Savarese, M.; Brémond, E.; Adamo, C. J. Phys. Chem. A 2016, 120, 1756.

[16] Ernzerhof, M.; Scuseria, G. E. J. Chem. Phys. 1999, 110, 5029.

[17] Adamo, C.; Barone, V. J. Chem. Phys. 1999, 110, 6158.

[18] Zhang, Y.; Xu, Goddard III, W. A. Proc. Natl. Acad. Sci. USA 2009, $106,4963$.

[19] Zhang, Y.; Wu, J.; Xu, X. Chem. Commun. 2010, 46, 3057.

[20] Zhang, Y.; Xu, X.; Jung, Y.; Goddard III, W. A. Proc. Natl. Acad. Sci. USA 2011, 108, 19896.

[21] Zhang, I. Y.; Su, N. Q.; Brémond, E.; Adamo, C.; Xu, X. J. Chem. Phys. 2012, 136, 174103. 
[22] Kümmel, S.; Perdew, J. P. Phys. Rev. B 2003, 68, 035103.

[23] Margraf, J. T.; Verma, P; Bartlett, R. J. J. Chem. Phys. 2016, 145, 104106.

[24] Smiga, S.; Franck, O.; Mussard, B.; Buksztel, A.; Grabowski, I.; Luppi, E.; Toulouse, J. J. Chem. Phys. 2016, 145, 144102.

[25] Perdew, J. P.; Burke, K.; Ernzerhof, M. Phys. Rev. Lett. 1996, 77, 3865.

[26] Brémond, E.; Adamo, C. J. Chem. Phys. 2011, 135, 024106.

[27] Brémond, E.; Sancho-García, J. C.; Pérez-Jiménez, A. J.; Adamo, C. J. Chem. Phys. 2014, 141, 031101.

[28] Brémond, E.; Savarese, M.; Sancho-García, J. C.; Pérez-Jiménez, A. J.; Adamo, C. J. Chem. Phys. 2016, 144, 124104.

[29] Jung, Y.; Lochan, R. C.; Dutoi, A. D.; Head-Gordon, M. J. Chem. Phys. 2004, 121, 9793.

[30] Neese, F. WIREs Comput. Mol. Sci. 2012, 2, 73.

[31] Lynch, B. J.; Truhlar, D. G. J. Phys. Chem. A 2003, 107, 8996.

[32] Yu, H. S.; Zhang, W.; Verma, P.; He, X.; Truhlar, D. G. Phys. Chem. Chem. Phys. 2015, 17, 12146.

[33] Haunschild, R.; Klopper, W. Theor. Chem. Acc. 2012, 131, 1112.

[34] Goerigk, L.; Grimme, S. J. Chem. Theory Comput. 2010, 7, 291.

[35] Stowasser, R.; Hoffmann, R. J. Am. Chem. Soc. 1999, 121, 3414.

[36] Meer, R.; Gritsenko, O. V.; Baerends, E. J. J. Chem. Theory Comput. 2014, 10, 4432. 
[37] Kim, M.-C.; Sim, E.; Burke, K. J. Chem. Phys. 2014, 140, 18A528.

[38] Goerigk, L.; Grimme, S. J. Chem. Theory Comput. 2011, 7, 291.

[39] Burke, K.; Cancio, A.; Gould, T.; Pittalis, S. arXiv:1409.4834 [condmat.mtrl-sci]

[40] Mori-Sánchez, P.; Cohen, A. J.; Yang, W. J. Chem. Phys., 2006, 125, 201102.

[41] Goerigk, L.; Grimme, S. J. Chem. Theory Comput. 2010, 6, 107.

[42] Cohen, A.; Mori-Sánchez, P.; Yang, W. Chem. Rev. 2012, 112, 289.

[43] Wykes, M.; Pérez-Jiménez, A. J.; Adamo, C.; Sancho-García, J. C. J. Chem. Phys. 2015, 142, 224105.

[44] Grimme, S.; Steinmetz, M. Phys. Chem. Chem. Phys. 2016, 18, 20926. 
- Table 1. Summary of the $\lambda_{x}$ and $\lambda_{c}$ values entering into the studied DH functionals.

- Table 2. Exchange and correlation energies $\left(E_{h}\right)$ for the atoms of the AE6 dataset. 
Table 1:

\begin{tabular}{lllll}
\hline Acronym & Year & $\lambda_{x}$ & $\lambda_{c}$ & $\left(1-\lambda_{c}\right)$ \\
\hline PBE0-DH & 2011 & $1 / 2$ & $1 / 8$ & $7 / 8$ \\
PBE-QIDH & 2014 & $3^{-1 / 3}$ & $1 / 3$ & $2 / 3$ \\
SOS1-PBE-QIDH & 2016 & $3^{-1 / 3}$ & $4 / 9$ & $2 / 3^{\mathrm{a}}$ \\
\hline
\end{tabular}

${ }^{a}$ Note the unexpected scaling due to the spinopposite-scaling construction of the model, see Ref. [6] for further details. 
Table 2:

\begin{tabular}{|c|c|c|c|c|c|c|}
\hline Energy $^{a}$ & & $\mathrm{H}$ & $\mathrm{C}$ & $\mathrm{O}$ & $\mathrm{Si}$ & $\mathrm{S}$ \\
\hline \multicolumn{7}{|l|}{$\left(1-\lambda_{x}\right) E_{x}^{\mathrm{PBE}}[\rho]$} \\
\hline & $\rho_{\mathrm{PBE}}$ & -0.0925 & -1.5384 & -2.4973 & -6.1745 & -7.6146 \\
\hline & $\rho_{\mathrm{PBE} 0}$ & -0.0929 & -1.5401 & -2.5003 & -6.1796 & -7.6196 \\
\hline & $\rho_{\mathrm{PBEH} \& \mathrm{H}}$ & -0.0933 & -1.5416 & -2.5032 & -6.1847 & -7.6246 \\
\hline & $\rho_{\mathrm{HF}-\mathrm{PBE}}$ & -0.0938 & -1.5427 & -2.5049 & -6.1886 & -7.6275 \\
\hline \multicolumn{7}{|l|}{$\lambda_{x} E_{x}^{\mathrm{EXX}}[\phi]$} \\
\hline & $\{\phi\}_{\mathrm{PBE}}$ & -0.2128 & -3.5033 & -5.6691 & -14.0370 & -17.3184 \\
\hline & $\{\phi\}_{\mathrm{PBE} 0}$ & -0.2140 & -3.5093 & -5.6795 & -14.0517 & -17.3331 \\
\hline & $\{\phi\}_{\text {PBEH\&H }}$ & -0.2152 & -3.5149 & -5.6894 & -14.0662 & -17.3474 \\
\hline & $\{\phi\}_{\mathrm{HF}-\mathrm{PBE}}$ & -0.2166 & -3.5200 & -5.6975 & -14.0788 & -17.3584 \\
\hline \multicolumn{7}{|l|}{$\left(1-\lambda_{c}\right) E_{c}^{\mathrm{PBE}}[\rho]$} \\
\hline & $\rho_{\mathrm{PBE}}$ & -0.0038 & -0.0959 & -0.1553 & -0.3224 & -0.3890 \\
\hline & $\rho_{\mathrm{PBE} 0}$ & -0.0039 & -0.0962 & -0.1560 & -0.3228 & -0.3894 \\
\hline & $\rho_{\mathrm{PBEH} \& \mathrm{H}}$ & -0.0039 & -0.0965 & -0.1565 & -0.3232 & -0.3898 \\
\hline & $\rho_{\mathrm{HF}-\mathrm{PBE}}$ & -0.0040 & -0.0962 & -0.1567 & -0.3228 & -0.3893 \\
\hline \multicolumn{7}{|l|}{$\lambda_{c} E_{c}^{\mathrm{PT} 2}\left[\phi, \phi^{\prime}\right]$} \\
\hline & $\left\{\phi, \phi^{\prime}\right\}_{\mathrm{PBE}}$ & -- & -0.0412 & -0.0756 & -0.0376 & -0.0704 \\
\hline & $\left\{\phi, \phi^{\prime}\right\}_{\mathrm{PBE} 0}$ & -- & -0.0345 & -0.0678 & -0.0307 & -0.0604 \\
\hline & $\left\{\phi, \phi^{\prime}\right\}_{\text {PBEH\&H }}$ & -- & -0.0301 & -0.0618 & -0.0261 & -0.0531 \\
\hline & $\left\{\phi, \phi^{\prime}\right\}_{\mathrm{HF}-\mathrm{PBE}}$ & -- & -0.0242 & -0.0530 & -0.0200 & -0.0427 \\
\hline
\end{tabular}

${ }^{\mathrm{a}} \lambda_{x}$ and $\lambda_{c}$ are those corresponding to the PBE-QIDH model. 
- Figure 1. Evolution of the MAD for the AE6 dataset, as calculated by the PBE0-DH, PBE-QIDH, and SOS1-PBE-QIDH using different sets of molecular orbitals.

- Figure 2. Evolution of the exchange and correlation components of the PBE-QIDH functional for several atoms using different sets of molecular orbitals. The plot for each atom at each subfigure has been shifted towards the value at $\lambda_{x}=0$ to ease the analysis.

- Figure 3. Evolution of the total exchange-correlation energy of the PBE-QIDH functional for several atoms using different sets of molecular orbitals. The plot for each atom has been shifted towards the value at $\lambda_{x}=0$ to ease the analysis.

- Figure 4. Evolution of the MAD for the SIE11 dataset, as calculated by the PBE0-DH, PBE-QIDH, and SOS1-PBE-QIDH using different sets of molecular orbitals. 


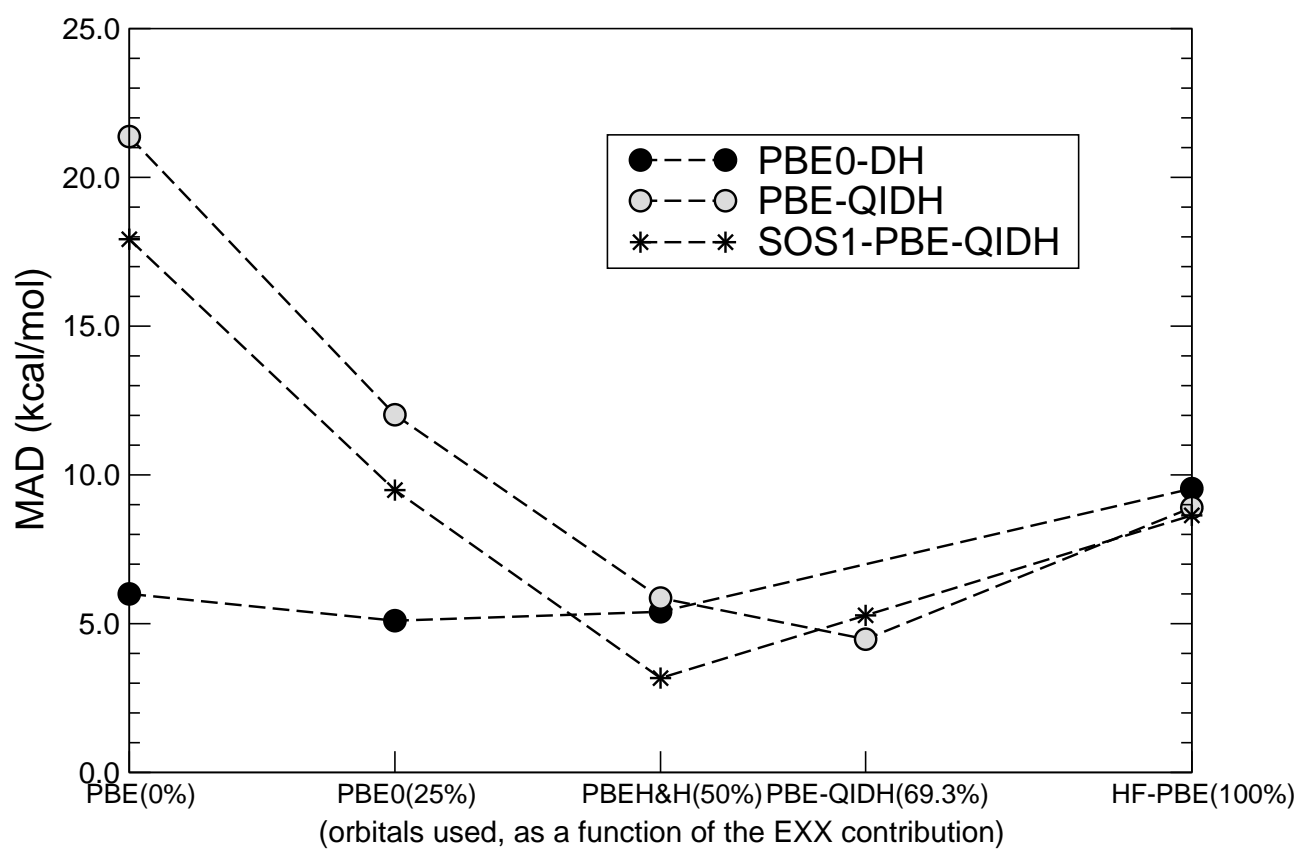

Figure 1. 


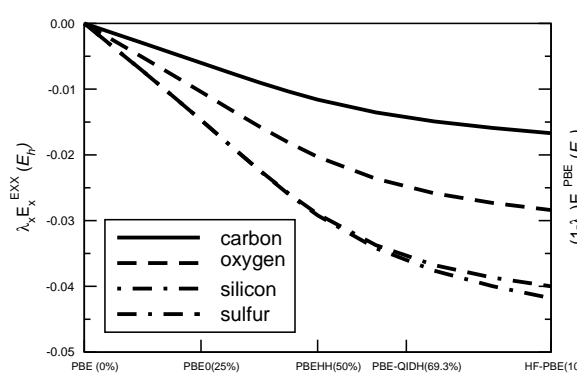

(orbitals used, as a function of the EXX contribution)

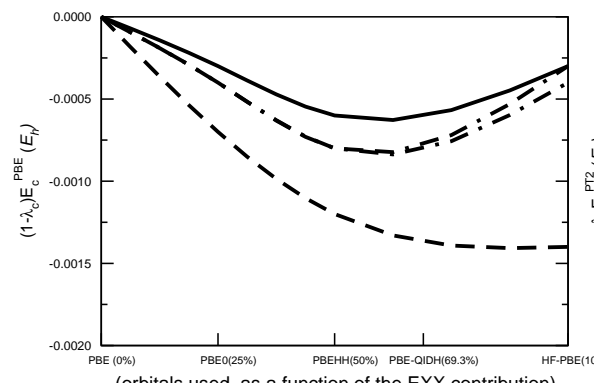

(orbitals used, as a function of the EXX contribution)

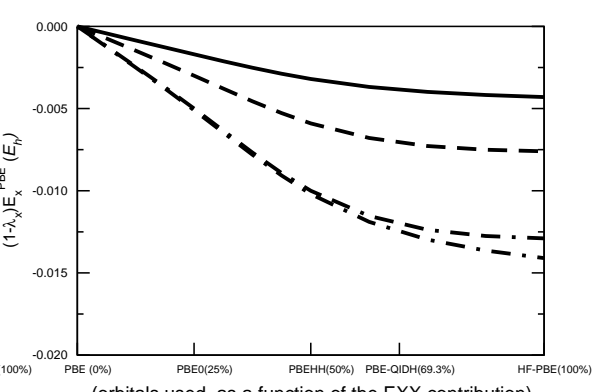

(orbitals used, as a function of the EXX contribution)

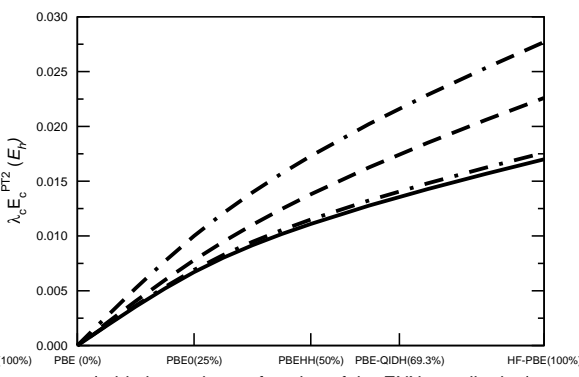

(orbitals used, as a function of the EXX contribution)

Figure 2. 


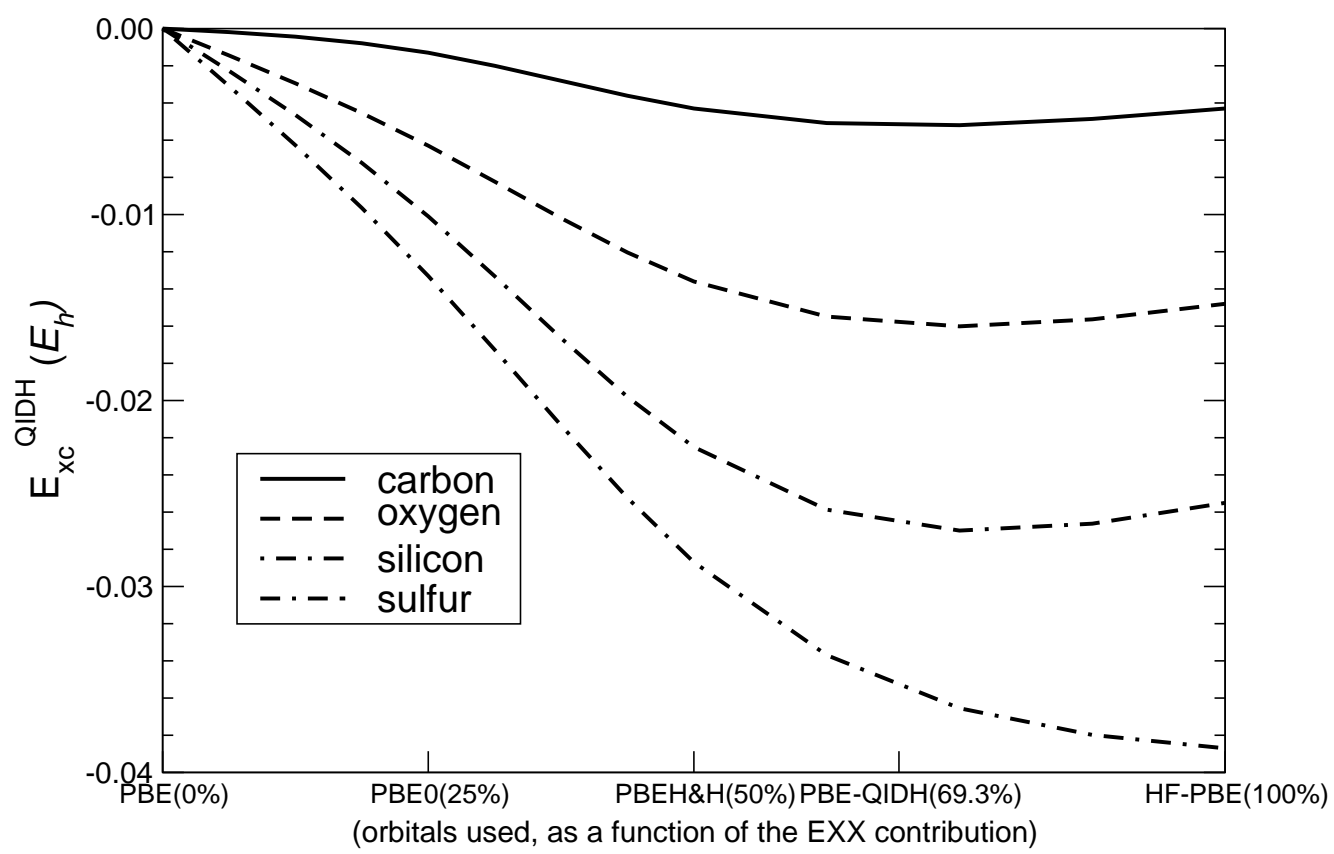

Figure 3. 


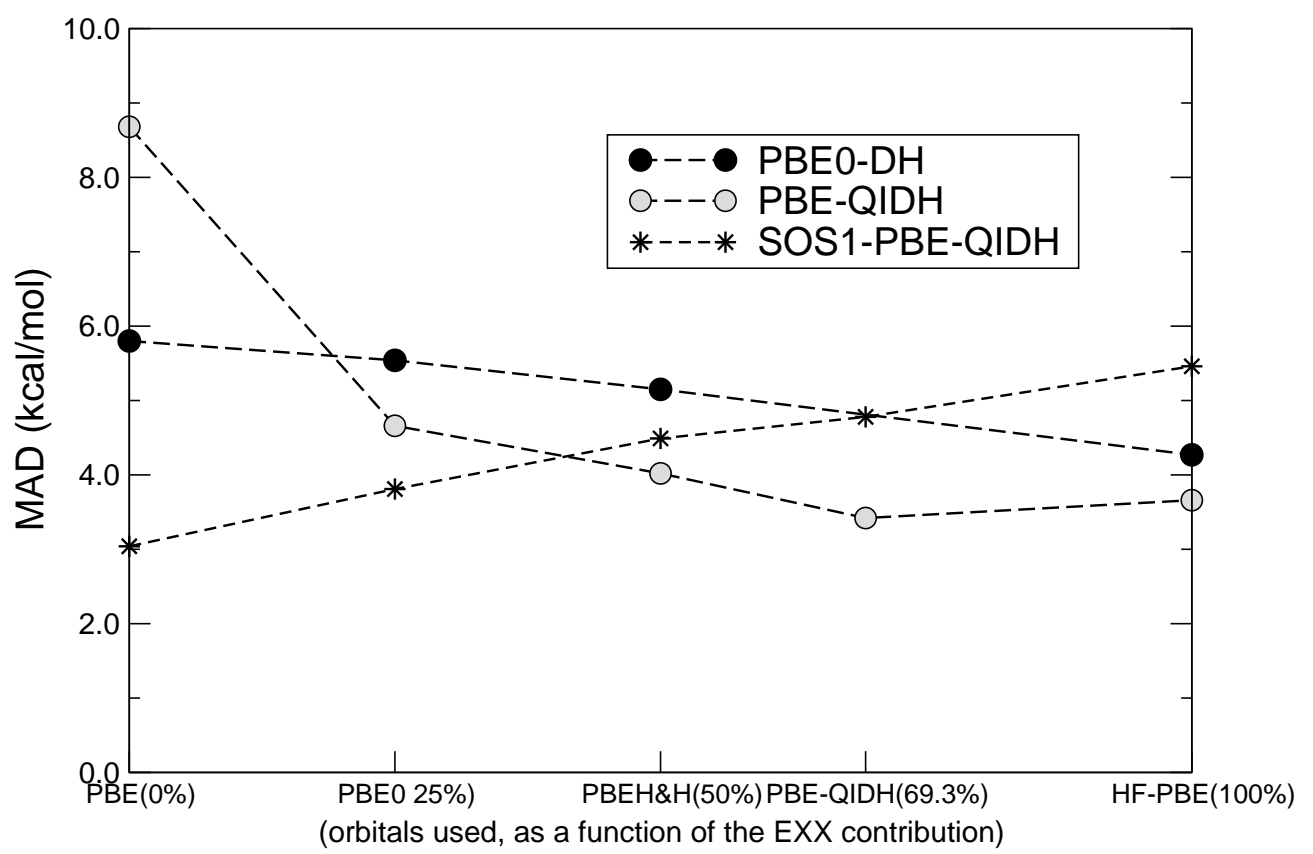

Figure 4. 